\title{
Pachyphytum confusum (Crassulaceae), una especie nueva del suroeste del estado de Guanajuato, México
}

\section{Pachyphytum confusum (Crassulaceae), a new species from the southwest of the state of Guanajuato, Mexico}

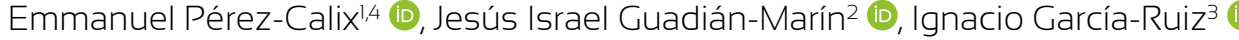

1 Instituto de Ecología, A.C., Red de Diversidad Biológica del Occidente Mexicano, Centro Regional del Bajío, Avenida Lázaro Cárdenas 253, Col. Centro, 61600 Pátzcuaro, Michoacán, México

2 Instituto de Ecología del Estado de Guanajuato, Aldana 12, Col. Pueblito de Rocha, 36040 Guanajuato, Guanajuato, México.

3 Instituto Politécnico Nacional, Centro Interdisciplinario de Investigación para el Desarrollo Integral Regional (CIIDIR) Unidad Michoacán, Justo Sierra 28, Col. Centro, 59510 Jiquilpan, Michoacán, México.

4 Autor para la correspondencia: emmanuel.perezcalix@inecol.mx

Recibido: 8 de junio de 2018. Revisado: 19 de junio de 2018. Aceptado: 29 de junio de 2018.

Primero en línea: 6 de agosto de 2018.

Publicado: 3 de octubre de 2018

\section{Citar como:}

Pérez-Calix, E., J. I. Guadián-Marín e I. García-Ruiz. 2018. Pachyphytum confusum (Crassulaceae), una especie nueva del suroeste del estado de Guanajuato, México. Acta Botanica Mexicana 125: 231-238. DOI: 10.21829/ abml25.2018.1401

$\mathrm{DOl}$

10.21829/abml25.2018.1401

\section{Resumen:}

Antecedentes y Objetivos: Pachyphytum (Crassulaceae) es un género endémico del centro de México; incluye plantas colgantes y suculentas que habitan riscos y taludes. La información taxonómica de Pachyphytum está incluida en obras generales de Crassulaceae y en una serie de artículos en los que se describen especies nuevas. A nivel regional, está contenida en el tratamiento de la familia Crassulaceae para la Flora del Bajío y de regiones adyacentes. El objetivo de este trabajo es nombrar y describir una especie nueva de Pachyphytum con base en plantas colectadas en el suroeste del estado de Guanajuato. Métodos: Se efectuaron excursiones a una de las localidades de donde se registró la planta en la Sierra de Pénjamo, y a la localidad tipo de Pachyphytum machucae. Se realizaron las descripciones de las plantas colectadas y se preparó un cuadro comparativo, se hizo una investigación en la literatura buscando especies semejantes morfológicamente.

Resultados clave: Se propone a Pachyphytum confusum como especie nueva para la ciencia, se compara con $P$. machucae y P. glutinicaule con las que muestra semejanzas morfológicas. Se le asigna el estatus de conservación de Vulnerable (VU).

Conclusiones: No obstante que Pachyphytum consta de 21 especies, interpretar sus relaciones ha sido complicado, en su última revisión se optó por considerar dos secciones, Pachyphytum y Diotostemon; la especie nueva se ubica en la primera. A los ejemplares de Pachyphytum colectados en la Sierra de Pénjamo, se les confundió hasta ahora con $P$. machucae, pero su comparación evidenció diferencias significativas para considerarlos entidades distintas. Otra especie con la que se asemeja, que presenta corolas más grandes que el cáliz, es Pachyphytum glutinicaule; sin embargo, presentan diferencias en los pétalos, en las brácteas y en las hojas. En algunas publicaciones se discute la inconsistencia de la clasificación infragenérica, criterio que se enfatiza con el descubrimiento de Pachyphytum confusum.

Palabras clave: Diotostemon, Pachyphytum machucae, Pachyphytum glutinicaule, Pénjamo.

\section{ABSTRACT:}

Background and Aims: Pachyphytum (Crassulaceae) is a genus endemic to central Mexico, consisting of hanging and succulent plants that grow on cliffs and slopes. The taxonomic information of $\mathrm{Pa}$ chyphytum is included in general works on Crassulaceae and a series of articles describing new species. At a regional level, it is included in the treatment of the family Crassulaceae for the Flora del Bajío y de regiones adyacentes. The aim of this work is to name and describe a new species of Pachyphytum based on plants collected in the southeast of the state of Guanajuato.

Methods: Excursions were realized to one of the localities in the Sierra de Pénjamo from where the plant was registered, as well as to the type locality of Pachyphytum machucae. Descriptions of the collected plants and a comparative table were prepared, and the literature was searched for morphologically similar species.

Key results: Pachyphytum confusum is proposed as a species new to science and is compared with $P$. machucae and $P$. glutinicaule which it resembles morphologically. The conservation status of Vulnerable (VU) is assigned.

Conclusions: Despite the fact that Pachyphytum consists of 21 especies, it is difficult to interprete their relationships; in its last revision two sections were considered; Pachyphytum and Diotostemon; the new species belongs to the first one. The specimens collected in the Sierra de Pénjamo were previously confused with $P$. machucae, but their comparison showed significant differences in order to consider them as a distinct species. Pachyphytum glutinicaule, another species which it resembles, has a corolla longer than the calyx; however, both species show differences in its petals, bracts and leaves. The inconsistency of the infrageneric classification is discussed in several publications, which is emphasized by the discovery of Pachyphytum confusum.

Key words: Diotostemon, Pachyphytum machucae, Pachyphytum glutinicaule, Pénjamo. 


\section{INTRODUCCIÓN}

Pachyphytum Link, Klotsch \& Otto (Crassulaceae) es un género endémico del centro de la República Mexicana (Pérez-Calix, 2008); así, 13 de sus 21 especies se conocen solo de la localidad donde se colectó el tipo. Los miembros del grupo son plantas colgantes, suculentas, de inflorescencias en cincino y de flores muy llamativas; son habitantes de riscos escarpados y taludes (Pérez-Calix, 2008).

El conocimiento taxonómico de Pachyphytum está incluido en dos obras generales de Crassulaceae, una a nivel mundial (Thiede, 2003) y otra de la familia en México (Meyrán y López-Chávez, 2003), en ambas se presenta la información de las 15 especies descritas hasta 2003; después se han descrito otras seis especies (García et al., 2002; Brachet et al., 2006; Reyes et al., 2007; Pérez-Calix et al., 2008; Meyrán, 2017; Pérez-Calix y Torres-Colín, 2018). Además de éstas, Kimnach (2010) propone ubicar en Pachyphytum a Echeveria cuicatecana Reyes, Pérez \& Brachet, a pesar de que en su publicación reconoce que los segmentos de la corola de esta última no presentan apéndices. Sin embargo, en el ejemplar tipo de la especie (J. Pérez Crisanto 584 (MEXU)), en la figura 5 del artículo de Kimnach (2010) y en la figura 5 de la descripción original (Reyes et al., 2004) se observa que los pétalos de E. cuicatecana son imbricados; características que diferencian a Pachyphytum de Echeveria DC. (Walther (1931) y Moran $(1963,1968)$.

La revisión de Pachyphytum del estado de Guanajuato está incluida en el tratamiento de la familia Crassulaceae para la Flora del Bajío y de regiones adyacentes (Pérez-Calix, 2008); en esta publicación se registró $P a$ chyphytum machucae I. García, Glass \& Cházaro (García et al., 1999), con base en plantas colectadas en la Sierra de Pénjamo, en el suroeste de Guanajuato (J. I. Guadián 137 (IEB)). Sin embargo, en una visita reciente de I. García al herbario IEB, notó que los ejemplares depositados en la colección del Instituto de Ecología, A.C., Centro Regional del Bajío, posiblemente no correspondían con la especie que él describió en coautoría con C. Glass y M. Cházaro. Considerado lo anterior, se procedió a colectar material en la localidad tipo de P. machucae y en la Sie- rra de Pénjamo. Una vez que se compararon los organismos, se concluyó que son especies diferentes, por lo que se decidió preparar esta publicación, con el objetivo de nombrar y describir una especie nueva de Pachyphytum con base en plantas colectadas en el suroeste del estado de Guanajuato.

\section{Materiales y Métodos}

La morfología de las plantas suculentas se modifica considerablemente en el proceso de herborización, por lo que es necesario trabajar con organismos vivos con fines de descripción y comparación. Por esa razón, para corroborar la identidad de la especie de Pachyphytum, colectada en la Sierra de Pénjamo, Guanajuato, se efectuó una excursión a una de las localidades de donde se registró previamente (ca. $6 \mathrm{~km}$ al SW de La Garita, 20³1'59.7'N, 10140'28.9”W, municipio Pénjamo, Guanajuato), y otra a la localidad tipo de Pachyphytum machucae (2 km al E de Pajacuarán, Barranca del Agua, cerca de El Cometa, municipio Pajacuarán, Michoacán).

Se prepararon las descripciones morfológicas de las plantas colectadas y se elaboró un cuadro comparativo (Cuadro 1), en el cual se observó que las discrepancias entre ambos organismos son tan importantes como para considerarlos especies diferentes. Además, se realizó una investigación bibliográfica y de herbario para corroborar si la planta de Guanajuato correspondía con alguno de los Pachyphytum ya conocidos. Después de concluir que no es ninguno de los publicados, se averiguaron las semejanzas morfológicas entre el Pachyphytum de Pénjamo con las otras especies, las características del taxon que se consideró más cercano se añadieron al Cuadro 1. Los ejemplares que se utilizaron para comparación se enlistan enseguida de los paratipos de la especie nueva.

\section{Resultados}

\section{Taxonomía}

Pachyphytum confusum Pérez-Calix, Guadián-Marín \& I. García, sp. nov. Fig. 1. 
Cuadro 1: Comparación morfológica de Pachyphytum confusum Pérez-Calix, Guadián-Marín \& I. García, P. glutinicaule Moran, y P. machucae I. García, Glass \& Cházaro.

\begin{tabular}{|c|c|c|c|}
\hline Carácter & $\begin{array}{l}\text { P. confusum Pérez-Calix, Guadián- } \\
\text { Marín \& I. García }\end{array}$ & P. glutinicaule Moran & $\begin{array}{c}\text { P. machucae I. García, Glass \& } \\
\text { Cházaro }\end{array}$ \\
\hline \multicolumn{4}{|l|}{ Hojas } \\
\hline forma & estrechamente elípticas & $\begin{array}{c}\text { obovadas a obovado-espatuladas u } \\
\text { oblanceoladas }\end{array}$ & elíptico-obovada \\
\hline $\operatorname{largo}(\mathrm{cm})$ & $4.2-7.2$ & $3-6.5$ & $4.2-6(7.5)$ \\
\hline ancho $(\mathrm{cm})$ & $1.3-2.2$ & $2-3.5$ & $1.4-1.6$ \\
\hline grueso $(\mathrm{cm})$ & $0.5-0.7$ & $0.3-1.5$ & 0.86 \\
\hline \multicolumn{4}{|l|}{ Brácteas florales } \\
\hline forma & elípticas & ovadas a elípticas & oblongo-elípticas \\
\hline largo (mm) & $7-10$ & $12-20$ & $5-18$ \\
\hline ancho (mm) & $4-6$ & $6-9$ & $2-5$ \\
\hline acomodo en el cincino joven & imbricadas & imbricadas & imbricadas \\
\hline Pedicelo (mm) & $4.5-6$ & $4-15$ & $3-5(10)$ \\
\hline Segmentos del cáliz & $\begin{array}{c}\text { 3.5-4 mm más cortos que la corola, } \\
\text { levemente desiguales en largo, } \\
\text { manifiestamente en ancho }\end{array}$ & $\begin{array}{l}\text { 2-6 mm más cortos que la corola, } \\
\text { desiguales largo y ancho }\end{array}$ & $\begin{array}{c}\text { iguales o ligeramente más largos } \\
\text { que la corola }\end{array}$ \\
\hline Segmentos largos forma & oblongos & $\begin{array}{l}\text { oblongos a angostamente triangular- } \\
\text { ovados }\end{array}$ & oblongo-oblanceolados \\
\hline largo $(\mathrm{mm})$ & $7.5-9$ & $8-15$ & $8-10$ \\
\hline ancho (mm) & $4-4.5$ & $5-7$ & $3-5$ \\
\hline Segmentos cortos forma & triangular & triangular-lanceolados & \\
\hline largo (mm) & ca. 7 & $6-9$ & $5-6$ \\
\hline ancho $(\mathrm{mm})$ & $2.5-3$ & $3-4$ & $2.2-2.5$ \\
\hline Segmentos de la corola & oblongo-lanceolados & oblongo-oblanceolados & oblongo-oblanceolados \\
\hline largo (mm) & $10-10.5$ & $12-17$ & $6-8$ \\
\hline ancho (mm) & ca. 4 & $3.5-5$ & $2-3$ \\
\hline color & blanco en la base, rojo en las dos & rojo & amarillo verdoso a verde pálido \\
\hline apéndice & $\begin{array}{l}\text { cuartas partes de su largo y verde } \\
\text { tenue en la zona apical } \\
\text { color rojo, formando parte de la } \\
\text { mancha roja del segmento de la } \\
\text { corola }\end{array}$ & rojo & rosa a rojizo \\
\hline
\end{tabular}

TIPO: MÉXICO. Guanajuato, municipio Pénjamo, ca. 6 km al SW de La Garita, 2010 m s.n.m., 20³1'59.7'”N, $101^{\circ} 40^{\prime} 28.9^{\prime}$ W, 7.II.2018, bosque tropical caducifolio en transición con bosque de encino, E. Pérez y J. I. Guadián 6749 (holotipo: IEB!, isotipos: CIMI!, MEXU!).
Plant suffrutescent, glabrous, stems erect or hanging and decumbent, up to $40 \mathrm{~cm}$ long; leaves agglomerated at the apex of the stems, narrowly elliptical, $4.2-7.2 \mathrm{~cm}$ long, 1.3-2.2 cm wide in the central part, 5-7 mm thick; inflorescence a cincinnus, peduncle up to $29 \mathrm{~cm}$ long, cincinnus 8-9 


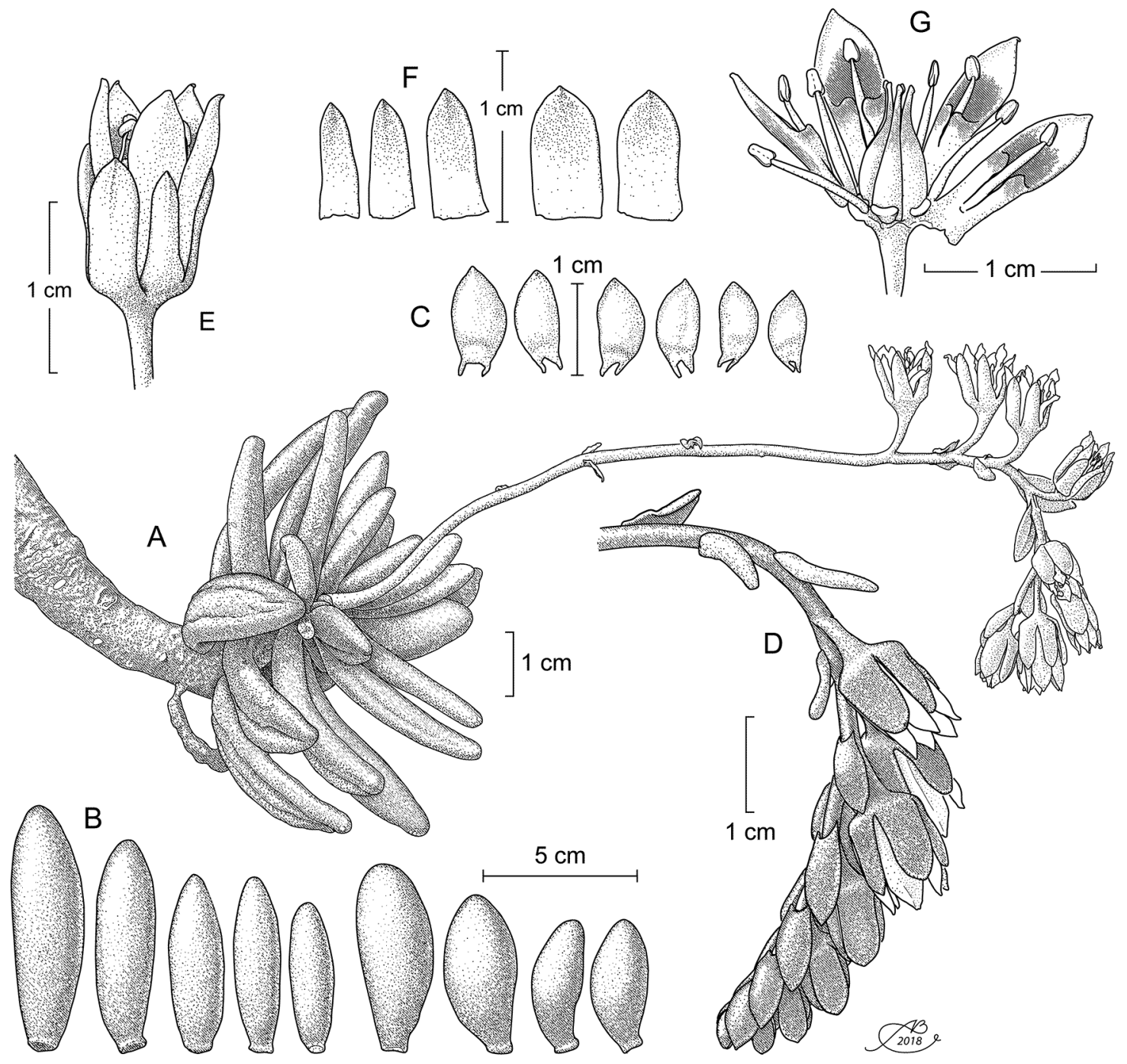

Figura 1: Pachyphytum confusum Pérez-Calix, Guadián-Marín \& I. García sp. nov. A. hábito; B. variación de las hojas; C. brácteas del cincino; D. vista lateral del cincino; E. vista lateral de la flor; F. variación de los segmentos del cáliz; G. disección de la flor. Con base en E. Pérez y J. I. Guadián 6749 (IEB, CIMI, MEXU). Ilustrada por Alfonso Barbosa.

cm long; bracts imbricate in young cincinni, elliptical, 7-10 $\mathrm{mm}$ long, 4-6 mm wide, apex acute, base sagittate; calyx 3.5-4 mm shorter than the corolla, lobes unequal in length and width; corolla subcampanulate, segments oblong-lanceolate, white at the base, pale green in the apical region, with a red spot on the central two quarters, appendices of the petals oblong, ca. $2 \mathrm{~mm}$ long, red; antisepalous stamens ca. $8 \mathrm{~mm}$ long, epipetalous stamens ca. $5.5 \mathrm{~mm}$ long; nectaries moon-shaped; ovary 3.5-4 mm high, styles $2.5-3.5 \mathrm{~mm}$ long, red; follicles ca. $1 \mathrm{~cm}$ long; seeds numerous.
Planta sufrutescente, suculenta, glabra, erecta o colgante y decumbente; tallos simples o ramificados cerca de la base, hasta $40 \mathrm{~cm}$ de largo, $2 \mathrm{~cm}$ de diámetro; hojas aglomeradas en el ápice del tallo, a veces formando una roseta laxa; láminas estrechamente elípticas en contorno, 4.2-7.2 cm de largo, 1.3-2.2 cm de ancho en la parte media, 5-7 mm de grueso, ápice romo, color verde pino, a veces café-rojizas en los márgenes y en el ápice, tornándose totalmente de ese color con la edad; inflorescencia en forma de cincino, pedúnculo hasta $29 \mathrm{~cm}$ de largo, 4-5 
$\mathrm{mm}$ de diámetro cerca de la base, cincino $8-9 \mathrm{~cm}$ de largo, con 7-12 flores; brácteas imbricadas en el cincino joven, 1 en la base de cada pedicelo, elípticas, 7-10 mm de largo, 4-6 mm de ancho, ápice agudo, base sagitada, color rosa; pedicelos 4.5-6 mm de largo, 1-1.6 mm de diámetro, color verde; cáliz 3.5-4 mm más corto que la corola, sépalos 5 , fusionados en la base, lóbulos brevemente desiguales en largo y evidentemente en ancho, 3 más anchos, 7.5-9 $\mathrm{mm}$ de largo, 4-4.5 mm de ancho, oblongos, agudos en el ápice, el más corto y angosto triangular, ca. $7 \mathrm{~mm}$ largo, 2.5-3 mm de ancho, ápice agudo, todos de color rosa en la base y verde olivo en las tres cuartas partes superiores; corola subcampanulada, exerta 3.5-4 $\mathrm{mm}$ del cáliz, pétalos 5 , connados ca. $1 \mathrm{~mm}$ en la base, segmentos oblongolanceolados, 10-10.5 mm de largo, ca. $4 \mathrm{~mm}$ de ancho en la parte media, ápice agudo, color blanco en la base, verde pálido en la región apical, con una mancha color rojo en las dos cuartas partes centrales, apéndices de los pétalos oblongos, ca. $2 \mathrm{~mm}$ de largo, color rojo; estambres antisépalos 5, filamentos ca. $6.5 \mathrm{~mm}$ de largo, anteras cerca 1.5 $\mathrm{mm}$ de largo, 5 epipétalos de ca. $3.5 \mathrm{~mm}$ de largo, anteras ca. $1 \mathrm{~mm}$, filamentos apicalmente de color rojo; nectarios lunulados, 1.5-2 mm de ancho, color amarillo claro; ovario $3.5-4 \mathrm{~mm}$ de alto, estilos $2.5-3.5 \mathrm{~mm}$ de largo, color rojo, estigmas capitados; folículos ca. $1 \mathrm{~cm}$ de largo; semillas numerosas, ca. $0.5 \mathrm{~mm}$ de largo, color café.

Distribución y hábitat: Pachyphytum confusum se conoce exclusivamente de algunas cañadas en la zona este de la Sierra de Pénjamo. La vegetación de la zona es bosque de encino en transición con bosque tropical caducifolio. Las especies arbóreas dominantes son Arbutus tessellata P.D. Sørensen, Clethra hartwegii Britton, $C$. mexicana DC., Lysiloma acapulcense (Kunth) Benth., Pistacia mexicana Kunth, Prunus serotina Ehrh., Quercus crassifolia Bonpl., Q. laurina Bonpl., Q. praeco Trel. y $Q$. praineana Trel.; algunos árboles alcanzan hasta 20 $\mathrm{m}$ de altura. Los estratos arbustivo y herbáceo se desarrollan principalmente cerca de las paredes de las cañadas o en lugares soleados. Las epífitas están representadas por Blechnum glandulosum Kaulf. ex Link, Diplazium lonchophyllum Kunze, Elaphoglossum petiolatum (Sw.) Urb., Laelia autumnalis (La Llave \& Lex.) Lindl., Peperomia tetraphylla Hook. \& Arn., Phlebodium areolatum (Humb. \& Bonpl. ex Willd.) J. Sm., Pleopeltis mexicana (Fée) Mickel \& Beitel y Tillandsia bourgaei Baker. En la región, las rocas son ígneas extrusivas y tobas riolíticas del Terciario Superior. En esta zona los promedios de las temperaturas son cercanas a $26.1{ }^{\circ} \mathrm{C}$ la máxima y a 12.9 ${ }^{\circ} \mathrm{C}$ la mínima; la precipitación promedio es de $656.8 \mathrm{~mm}$ anuales (Guadián-Marín, 2013).

Fenología: Pachyphytum confusum es una planta sufrutescente, suculenta y perennifolia, con hojas carnosas. Subsiste la época desfavorable en estado vegetativo, en el que se aprecia en los riscos, ya sea colgante o erecta; florece de diciembre a marzo.

Estado de conservación: Pachyphytum confusum se conoce de tres subpoblaciones conformadas por pocos individuos que habitan en la porción oriental de la Sierra de Pénjamo, en Guanajuato. Aunque los parajes en los que se establece son de difícil acceso, lo que sugiere alguna protección a la extracción, se le asigna en la categoría de Vulnerable, por cumplir el Criterio D, subcriterios 1 y 2 (VU D1 + 2), de la propuesta de la International Union for Conservation of Nature (IUCN, 2012). Se estima que en las subpoblaciones que se conocen suman menos de 1000 individuos maduros y su área de ocupación es muy reducida (ca. $0.27 \mathrm{~km}^{2}$ ).

Etimología: el epíteto específico es del latín confusus, confundido, hace alusión a que, en un principio, las plantas se habían confundido con P. machucae.

Material adicional examinado (paratipos): MÉXICO. Guanajuato, municipio Pénjamo, Cañada, $2060 \mathrm{~m}$ s.n.m., bosque de encino, 7.X.2006, J. I. Guadián 124 (IEB); 6 km al SW de La Garita, 2132 m s.n.m., 7.X.2006, J. I. Guadián 137 (IEB); $9 \mathrm{~km}$ al W de La Garita, cerca de la peña de La Iglesia, 2150 m s.n.m., bosque de galería, 20.I.2010, J. I. Guadián 530 (IEB). 
Ejemplares revisados de Pachypytum glutinicaule Moran: MÉXICO. Aguascalientes, municipio Calvillo, cañada cercana a Puentes Cuates rumbo a Río Gil, 2000 m s.n.m., 21.V.1997, G. García 3871 (IEB). Querétaro, municipio Cadereyta, Tziquia, frente a La Sabina, 1530 m s.n.m., 30.III.1993, S. Zamudio 9013 (IEB). Municipio San Joaquín, cañada La Culebra, al NE de La Tinaja, 1570 m s.n.m., matorral submontano, 8.III.1996, S. Zamudio et al. 9772 (IEB).

Ejemplares revisados de Pachypytum machucae I. García, Glass \& Cházaro: MÉXICO. Michoacán, municipio Pajacuarán, $2 \mathrm{~km}$ al E de Pajacuarán, Barranca del Agua, cerca de El Cometa, 1850 m s.n.m., bosque tropical caducifolio, 7.I.1997, I. García Ruiz 4497 (holotipo: IEB, isotipo CIMI); $0.5 \mathrm{~km}$ al SE de Pajacuarán, $1740 \mathrm{~m}$ s.n.m., bosque tropical caducifolio, 1.III.1999, I. García Ruiz 5566 (IEB); aprox. $0.5 \mathrm{~km}$ al E-SE de Pajacuarán, 1740 m s.n.m., bosque tropical caducifolio, 1.IV.1999, I. García Ruiz 6289 (CIMI); aprox. $1 \mathrm{~km}$ al E-SE de Pajacuarán, Sierra de Pajacuarán, 1850 m s.n.m., matorral subtropical, 15.XI.2011, I. García Ruiz 8435 (CIMI); aprox. 1 km al E-SE de Pajacuarán, Sierra de Pajacuarán, 1780 m s.n.m., matorral subtropical, 29.I.2018, I. García Ruiz y J. Méndez 9295 (CIMI, IEB).

\section{Discusión}

El género Pachyphytum consta de solo 21 especies, no obstante, interpretar las relaciones entre ellas ha sido complicado. Berger en 1930 lo dividió en dos grupos, $\mathrm{Pa}$ chyphytum y Diotostemon, a los que Walther en 1931 les asignó el rango de secciones; en esta época se conocían solo 10 especies. Moran (1968) observó que dos miembros, uno descrito por él (Moran, 1963) y otro por Britton y Rose (1905) no correspondían con ninguno de los grupos, por lo que propuso la sección Ixiocaulon, misma que enmendó después para asignar un nuevo taxon (Moran, 1968; 1971). El hallazgo y descripción de otros elementos han puesto de manifiesto lo artificial de la clasificación infragenérica (García et al., 1999, 2002; PérezCalix y Glass, 1999). Con fundamento en lo anterior, Thiede (2003) optó por considerar solo dos secciones,
Pachyphytum y Diotostemon. Con base en este criterio, Pachyphytum confusum se ubica en la primera, por el siguiente juego de caracteres:

- hojas dispuestas laxamente, sin tocarse entre ellas

- láminas foliares \pm planas, la relación ancho/grueso es mayor que dos

- brácteas del cincino sagitadas y amplexicaulas en la base, imbricadas en la inflorescencia joven

- $\quad$ segmentos del cáliz marcadamente desiguales en dimensiones y forma, tres mayores y dos pequeños

- $\quad$ segmentos de la corola ligeramente recurvados al final de la antesis

- pétalos de color blanco con una mancha roja en la región media ventral y cubriendo las escamas petaloides

- filamentos apicalmente de color rojo

Precedentemente a los ejemplares de Pachyphytum colectados en la Sierra de Pénjamo se les asignó el nombre P. machucae por las semejanzas vegetativas y algunas relativas a la inflorescencia y a la flor como la forma y dimensiones de las hojas, apariencia general del cincino y patrón de coloración de los pétalos. Sin embargo, la comparación detallada con material fresco evidenció que se diferencian en la forma y ancho de las brácteas florales, la relación de largo cáliz/corola, el largo de los lóbulos del cáliz y los segmentos de la corola, y el color de los sépalos (Cuadro 1).

De otras especies de la sección Pachyphytum, la única que presenta corolas más grandes que el cáliz es $P a$ chyphytum glutinicaule; sin embargo, a diferencia de $\mathrm{Pa}$ chyphytum confusum sus pétalos son color rosa oscuro, uniformemente coloreados u oscureciéndose ventralmente y con los ápices ligeramente más claros. Por lo demás, las diferencias entre las dos especies incluyen características de las láminas foliares (forma y ancho), de las brácteas florales (forma y dimensiones), del cáliz y la corola (Cuadro 1).

\section{CONCLUSIONES}

La sospecha de que un ejemplar de herbario no está correctamente identificado, así como la necesidad de realizar exploraciones para asignarle nombre a una crasulácea, 
enfatiza la importancia de disponer de plantas frescas, en el caso de las suculentas, para identificarlas y describirlas previo a su secado; también es recomendable tomar una serie de fotografías en campo y en laboratorio.

Después de las publicaciones de Meyrán y LópezChávez (2003) y de Thiede (2003), se han descrito cinco especies de Pachyphytum, en algunas de estas publicaciones se discute la inconsistencia de la clasificación infragenérica, así como la necesidad de realizar investigaciones que nos permitan vislumbrar la filogenia del grupo. Esa insinuación se enfatiza con el descubrimiento de $\mathrm{Pa}$ chyphytum confusum ya que su ubicación en la sección Pachyphytum origina que se altere la definición del grupo.

\section{CONTRIBUCIÓN DE AUTORES}

EPC y JIG realizaron la exploración en la Sierra de Pénjamo, e IGR la de Sierra de Pajacuarán. Los tres autores participaron en la elaboración del cuadro comparativo. EPC preparó el manuscrito con ayuda de JIG e IGR. Todos los autores contribuyeron a la discusión, revisión y aprobación del manuscrito final.

\section{FINANCIAMIENTO}

La investigación se generó con financiamiento del Instituto de Ecología, A.C. (proyecto 20006-10496) y del Instituto Politécnico Nacional a través de los apoyos de COFAA y de EDI.

\section{AgRADECIMIENTOS}

Agradecemos a Patricia Y. Mayoral Loera y a MarieStéphanie Samain la lectura crítica del manuscrito; además, a Marie-Stéphanie le reconocemos su apoyo en la traducción del resumen y del protólogo de la especie al inglés. La ilustración es obra de Alfonso Rafael Barbosa García. A José Méndez le reconocemos su apoyo en campo en la colecta de Pachyphytum machucae.

\section{LITERATURA CITADA}

Berger, A. 1930. Crassulaceae. In: Engler, A. y K. Prantl (eds.). Die Natürlichen Pflanzenfamilien. vol. 18a. 2 ed. Verlag Wilhelm Engelmann. Leipzig, Germany. Pp. 352-458.
Brachet, I. C., J. Reyes-Santiago y R. Mondragón. 2006. Pachyphytum saltensis Brachet, Reyes \& Mondragón, una nueva especie para el estado de Zacatecas, México. Cactáceas y Suculentas Mexicanas 51(2): 46-51.

Britton, N. L. y J. N. Rose. 1905. Crassulaceae. North American Flora 22: 7-74.

García, R. I., C. Glass y M. Cházaro. 1999. Pachyphytum machucae (Crassulaceae) una nueva especie de Michoacán, México. Acta Botanica Mexicana 47: 9-14. DOI: https://doi.org/10.21829/abm47.1999.820

García, R. I., E. Pérez-Calix y J. Meyrán G. 2002. Especie nueva de Pachyphytum (Crassulaceae) del oriente de Michoacán, México. Anales del Instituto de Biología, Universidad Nacional Autónoma de México, Serie Botánica 73(2): 147-253.

Guadián-Marín, J. I. 2013. Flora y Vegetación de la Sierra de Pénjamo, Guanajuato (México). Tesis de licenciatura. Facultad de Biología, Universidad Michoacana de San Nicolás de Hidalgo. Morelia, Michoacán, México. 147 pp.

IUCN. 2012. IUCN Red List Categories and criteria: Version 3.1. 2nd ed. Gland, Switzerland and Cambridge, UK. iv $+32 \mathrm{pp}$.

Kimnach, M. 2010. Notes on Crassulaceae: 2. Cactus and Succulent Journal (US) 82: 122-125.

Meyrán, G. J. 2017. Pachyphytum neglectum sp. nov. Meyrán. Cactus-Aventures International 2-2017: 38-41.

Meyrán, G. J. y L. López-Chávez. 2003. Las Crassuláceas de México. Sociedad Mexicana de Cactología, A.C. México, D.F., México. 234 pp.

Moran, R. 1963. Pachyphytum brevifolium Rose and Pachyphytum glutinicaule, a new species from Hidalgo, México. Cactus and Succulent Journal (US) 15: 35-41.

Moran, R. 1968. New subgeneric groups in Echeveria and Pachyphytum. Cactus and Succulent Journal (US) 40: 3642.

Moran, R. 1971. Pachyphytum fittkaui, a new species from Guanajuato, Mexico. Cactus and Succulent Journal (US) 43: 26-32.

Pérez-Calix, E. 2008. Familia Crassulaceae. Flora de Bajío y de regiones adyacentes 115: 1-141. 
Pérez-Calix, E. y C. Glass. 1999. Pachyphytum brevifolium Rose (Crassulaceae) a un siglo de su descubrimiento y Pachyphytum garciae, una especie nueva del centro de México. Acta Botanica Mexicana 48: 1-10. DOI: https:// doi.org/10.21829/abm48.1999.829

Pérez-Calix, E. y R. Torres-Colín. 2018. Pachyphytum rogeliocardenasii (Crassulaceae), a new species from northwestern Querétaro, México. Phytotaxa 348(1): 5662. DOI: https://dx.doi.org/10.11646/phytotaxa.348.1.7

Pérez-Calix, E., I. García R. y M. Cházaro B. 2008. Una especie nueva de Pachyphytum (Crassulaceae) para la flora de Jalisco, México. Acta Botanica Mexicana 83: 63-68. DOI: https://doi.org/10.21829/abm83.2008.1061
Reyes, S. J., O. González Z. y A. Gutiérrez de la Rosa. 2007. Pachyphytum brachetii, una nueva especie del estado de Hidalgo, México. Cactáceas y Suculentas Mexicanas 52(2): 53-63.

Reyes, S. J., J. Pérez y C. Brachet. 2004. Echeveria cuicatecana, una nueva especie para el estado de Oaxaca, México. Cactáceas y Suculentas Mexicanas 49(3): 80-84.

Thiede, J. 2003. Pachyphytum. In: Eggli, U. (ed.). Crassulaceae. Illustrated handbook of succulent plants. Springer. Berlin, Germany. Pp. 190-195.

Walther, E. 1931. Genus Pachyphytum. Journal of the Cactus and Succulent Society of America 3: 9-13. 